5 Tyrer P. The end of the psychopharmacological revolution. Br J Psychiatry 2012: 201: 168.

Dr David Shiers, MB ChB, MRCP (UK), retired GP, Leek, North Staffordshire, former joint lead on National Early Intervention in Psychosis programme of National Mental Health Development Unit (2004-2010). Professor Sue Bailey, MBChB, FRCPsych, President of the Royal College of Psychiatrists, consultant child and adolescent forensic psychiatrist, Greater Manchester West Mental Health NHS Foundation Trust, and Professor of Child and Adolescent Mental Health, University of Central Lancashire. Dr Clare Gerada, FRCP, FRCGP, FRCPsych, Chair of Council, Royal College of General Practitioners, and GP partner, Hurley Clinic, London. Professor Helen Lester, MB ChB, MA, MD, FRCGP, Professor of Primary Care, Birmingham University.

doi: $10.1192 / p b .37 .1 .35$

\section{A case of clozapine-induced diabetic ketoacidosis}

A 29-year-old male of Yemeni descent detained in a medium secure unit was commenced on clozapine; after 4 weeks of treatment he was taking a total of $275 \mathrm{mg}$ in divided doses. He developed nausea and vomiting which progressed over 36 hours to a point where he needed to be urgently transferred to the local accident and emergency unit. At assessment he was experiencing breathing problems, vomiting and he was incontinent of urine; he had a Glasgow Coma Scale score of five. He was immediately transferred to the intensive care unit. The differential diagnoses included drug overdose, alcohol intoxication and clozapine-induced hyperglycaemia. His blood chemistry showed evidence of diabetic ketoacidosis; his blood glucose level was grossly elevated. The clozapine was stopped and the patient was given appropriate treatment with glycaemic agents.

In summary, the patient had become seriously unwell over a period of 36 hours. Apart from having a slightly raised body mass index, he was fit and well and had no family history of diabetes. His pre-treatment blood glucose had been normal.

Diabetic ketoacidosis is over ten times more common in patients treated with atypical antipsychotics than in the general population, ${ }^{1}$ although the evidence is largely restricted to case reports and series. ${ }^{2}$ Clozapine has a higher risk of ketoacidosis than other oral antipsychotics ${ }^{3}$ and it tends to develop after a shorter duration of treatment, with a high proportion of patients developing it within 3-6 months. Low doses, being a young male and having a negative family history seem to be significant risk factors. ${ }^{4}$ There is also significant mortality. ${ }^{5}$ The unusual aspect of this case (although not unknown) was the occurrence of diabetic ketoacidosis during the titration phase of treatment.

1 Henderson DC, Cagliero E, Copeland PM, Louie PM, Borba CP, Fan X, et al. Elevated haemoglobin A1c as a possible indicator of diabetes mellitus and diabetic ketoacidosis in schizophrenia patients receiving atypical antipsychotics. J Clin Psychiatry 2007; 68: 533-41.

2 Jin $\mathrm{H}$, Meyer JM, Jeste DV. Phenomenology of and risk factors for new onset diabetes mellitus and diabetic ketoacidosis associated with atypical antipsychotics: an analysis of 45 published cases. Ann Clin Psychiatry 2002; 14: 59-64.

3 Leslie DL, Rosenheck RA. Incidence of newly diagnosed diabetes attributable to atypical antipsychotic medications. Am J Psychiatry 2004; 161: 1709-11.

4 Nihalani ND, Tu X, Lamberti JS, Olson D, Olivares T, Costea GO, et al. Diabetic ketoacidosis among patients receiving Clozapine: a case series and review of socio-demographic risk factors. Ann Clin Psychiatry 2007; 19: $105-12$.
5 Koller E, Schneider B, Bennett K, Dubitsky G. Clozapine-associated diabetes. Am J Med 2011; 111: 716-23.

Yasir Kasmi, Consultant Forensic Psychiatrist, Partnerships in Care, UK, email: yasir.kasmi@partnershipsincare.co.uk

doi: $10.1192 / p b .37 .1 .36$

\section{'Deaf-mute': time to abandon stigmatisation of the deaf community}

I was dismayed to read Akintomide et al's reference to the subject of their case review as a person who was 'profoundly deaf-mute'.

'Deaf-mute' is an outdated term originating in the 18th/ 19th century. It carries very derogatory connotations, and is no longer used in reference to individuals with profound deafness. The term 'mute' implies a lack of ability to make noise. Such a label is technically inaccurate when applied to deaf individuals, since they generally have functioning vocal chords and therefore retain the ability to make vocalisations (http:// wfdeaf.org). Those who are profoundly deaf from early life struggle to develop an oral language, given that hearing is required to facilitate a modulation of one's voice into speech. Many will therefore employ non-verbal communication in the form of sign language instead. This is a complex combination of hand signals, with its own regional dialects and international differences.

Over 75000 people in Britain currently use British Sign Language (BSL) as their first or preferred language. The majority of these sign language users consider themselves as members of a distinct cultural community with a strong social identity. $^{2}$

To this day the social image of deafness remains impaired on an international scale. This manifests itself in the form of a deeply rooted pathological stigma, negative stereotypes and prejudiced attitudes towards the deaf. ${ }^{3}$ It would seem that such ignorance also persists among health professionals. Ralston et $a l^{4}$ surveyed the attitudes of 165 physicians and identified a significant difference in attitudes towards hearing patients compared with deaf patients. Munoz-Baell \& Ruiz ${ }^{3}$ suggest that much of the stigma relating to the deaf community arises from an extensive social lack of appreciation of both their communication mechanisms and their culture. Unfortunately, in spite of more recent advances in healthcare legislation, ${ }^{5}$ it would appear that there is still some way to go before members of the deaf community achieve the equality of health and social standing to which they are entitled.

The summary for Akintomide et al's paper states that it is the first published case report of catatonia in someone who is profoundly deaf. It is a shame therefore that, rather than taking the opportunity to present a positive reflection of managing patients with profound deafness, the authors have merely succeeded in perpetuating existing negative stereotypes about this sector of the population.

$\mathrm{Nb}$. Deaf is used in reference to those born deaf whose first language is BSL. It is used as a generic term, and for those with acquired deafness whose primary form of communication is oral.

1 Akintomide GS, Williams Porter S, Pierce A. Catatonia in a woman who is profoundly deaf-mute: case report. Psychiatrist 2012; 36: 418-21. 
2 Adshead S, du Feu M. Mental health service provision for the deaf community. Progress Neurol Psychiatry 2005; 9: 26-30.

3 Munoz-Baell IM, Ruiz MT. Empowering the deaf. Let the deaf be deaf. $J$ Epidemiol Comm Health 2000; 54: 40-4.

4 Ralston E, Zazove P, Gorenflo DW. Physicians' attitudes and beliefs about deaf patients. J Am Board Fam Practice 1996; 9: 167-73.

5 Department of Health. Mental Health and Deafness - Towards Equity and Access: Best Practice Guidance. Department of Health, 2005.

Sara L. Adshead, Consultant Psychiatrist (formerly locum consultant psychiatrist), National Deaf Mental Health Service, Leamington Spa, Warwickshire, UK, email: sara.adshead@covwarkpt.nhs.uk

doi: $10.1192 / \mathrm{pb} .37 .1 .36 \mathrm{a}$

\section{The impact of the Health and Social Care Act 2012 on forensic psychiatry}

As a National Health Service forensic psychiatrist working on a newly commissioned low secure ward, the statement: 'it is all too predictable that yet more patients will be pushed down forensic care pathways from which return to mainstream care will be difficult (p. 402)' in Holloway's excellent November editorial $^{1}$ struck a firm chord with me.

In the past year, I have overseen an expansion of both the low secure forensic estate and the out of area patient placements. Although there was some clinical and commissioning intent to introduce the low secure estate to allow transition out of the medium secure estate (and indeed this has happened to some extent), there has been quite a surge of patients coming from the general acute services and the community.

We also receive some prison transfers; these include general adult community patients with no prior forensic history who were missed in the community owing to (poorly resourced) service lapses. Such patients become 'forensic' because of a lack of adequate community psychiatric services rather than being appropriate referrals to the service. In any case, we are expanding.

Good news for forensic staff, but not so good for patient care. Earlier psychiatric intervention for them may have even saved them from being locked up in prison. This is low-income country psychiatry in a high-income country.

At a recent presentation by some Californian psychiatrists, I was very impressed by the vigour with which they grapple with often very difficult legal circumstances of psychiatric care in their jurisdiction. They noted that most of their state hospital beds were occupied by their forensic patients. There was very little available for non-forensic patients, either in hospital or in the community. I wonder whether here in England we are also heading in that direction.

Finally, it appears that in this evolving, risk-focused, forensic-heavy psychiatric care environment, the 'forensic' patient today is not the same forensic patient from 20 years ago. These days, not every forensic patient is a high secure step-down patient. Why is it then more difficult to discharge forensic patients into the community, and return them to mainstream services? At the very least, the expanding low secure estate ought to provide an easier interface within the psychiatric services than was the case in the past. This way we will have done our best for our patients while contending with the difficult care environment being planned for us by this government. Indeed, who else will?

1 Holloway F. The Health and Social Care Act 2012: what will it mean for mental health services in England? Psychiatrist 2012; 36: 401-3.

Muzaffar Husain, Consultant Forensic Psychiatrist, Kent Forensic Psychiatric Service, Beckenham, Kent, UK, email is Muzaffar.Husain@ kmpt.nhs.uk

doi: $10.1192 / p b .37 .1 .37$

\section{Needless complexity in commissioning}

Having attended a local third-sector and service user conference and having read the editorial by Holloway, ${ }^{1}$ I wonder whether the following needs more consideration.

It strikes me that dividing mental health commissioning responsibilities locally between the clinical commissioning groups (mental illness treatments) and local authorities (suicide and substance misuse prevention, mental health promotion) poses unnecessary complexity and bureaucratic waste. Despite lay representation in clinical commissioning groups, there is no democratic accountability similar to that offered through local councillors and local authority scrutiny committees which can call providers to attend a public meeting to account for their priorities in using public funds. Perhaps local elections might be more popular if electors realise that councillors could be voted out if they are not active in championing mental health issues such as dementia care. Furthermore, local authorities already have experienced procurement teams with ready access to performance management and audit functions.

Therefore, I wonder whether clinical commissioning groups should be relieved of all mental health commissioning responsibilities, with this function carried out entirely by local authorities. This would allow the commissioning groups to concentrate on acute and chronic medical diseases (which contribute to most of the cost via hospital bed usage and new technology). The added benefit of mental health being commissioned by local authorities would be integration of social and healthcare budgets for the benefit of people with severe mental illness such as psychosis and dementia. As a practising clinician, I find it difficult to separate social and health interventions in providing a good outcome for an individual patient; usually, there is a synergistic effect.

The other issue discussed by Holloway is 'personalisation'. It is hoped that by April 2013, 70\% of eligible mental health service users (mainly with severe chronic illness) will have a personal budget with an allocated broker to help clarify and achieve their choices in interventions. The above rationalising of commissioning would lend itself to a combined health and social care budget which can be spent pragmatically. A chipand-pin charge card could be introduced to carry a combined budget, with greater accountability and freedom from having to collect receipts.

The third issue highlighted at the conference was an increasing body of evidence suggesting that active collaborations between statutory mental health providers and third-sector organisations result in better outcomes and lower number of bed days in psychiatric hospitals. Perhaps this should be considered an essential requirement for mental health trusts when submitting bids for a service. 